\title{
Based on the ANSYS double rhombic honeycomb paperboard finite element analysis
}

\author{
Baozhong Wang \\ Hebei United University, No.46, Xinhua West Road, \\ Tang Shan City, Hebei Province, China \\ Wangbzhong666@sohu.com
}

\author{
Shan Gao \\ Hebei United University, No.46, Xinhua West Road, \\ Tang Shan City, Hebei Province, China \\ 582934067@qq.com
}

\begin{abstract}
The bearing capacity of the structure of honeycomb paperboard and its performance has the direct relations. In order to design a rational structure, good performance, good economic performance of honeycomb paperboard, using finite element analysis method, the use of ANSYS finite analysis software to establish the model of analysis of bilayer honeycomb paperboard, double diamond static properties of honeycomb paperboard, proved that the double rhombic honeycomb paperboard compression performance of honeycomb paperboard, for improved structure and performance the increase will have a certain reference.
\end{abstract}

Keywords- honeycomb paperboard; static characteristics; ANSYS

\section{INTRODUCTION}

Honeycomb board as a lightweight structural materials, early honeycomb aluminum is widely used in aviation industry. The honeycomb paperboard is a kind of new green renewable materials, it not only makes the plate overall mechanical properties are improved, but also causes the plate stiffness and improved stability ${ }^{[1]}$. Honeycomb paperboard has the advantages of high strength, lightweight, big bearing capacity, transport is the most important environmental protection and have certain buffer capacity of packaging material, it can guarantee the products in the transport of the security ${ }^{[2-3]}$. The Institute of packaging and enterprises put the honeycomb paperboard research as a focus and hot, except that it has a good application prospect, is also a very high commercial value. Wang Mei through the static compression tests the cushioning property of honeycomb paperboard was studied by ${ }^{[4]}$. Zhang Anning on the multilayer superimposition honeycomb cardboard compression characteristics are analyzed and the different rate under static compression test. This paper mainly based on ANSYS to build the finite element model of bilayer honeycomb paperboard, stress strain analysis, and through discussing the analysis result, some valuable conclusions are obtained.

\section{MODEL ESTABLISHMENT}

ANSYS 13 software mainly provides the following kinds of model: modeling, solid modeling, input externally created finite element model. By double rhombic honeycomb paperboard structure characteristics, modeling method using a bottom-up approach, first create the key point, connected into the line after stretching into the surface, then from a point to a line by line to surface, from elementary to advanced model. And then through the mirror copy command to get double rhombic honeycomb core structure; as a result of honeycomb paperboard honeycomb paperboard paper thickness relative to the thickness is very thin, so it can be to double rhombic honeycomb cardboard stress model for the following simplified to study its mechanical model ${ }^{[5-6]}$, can be viewed as a film, simplified ANSYS after the establishment of the honeycomb paper core physics model as shown in figure 1 .

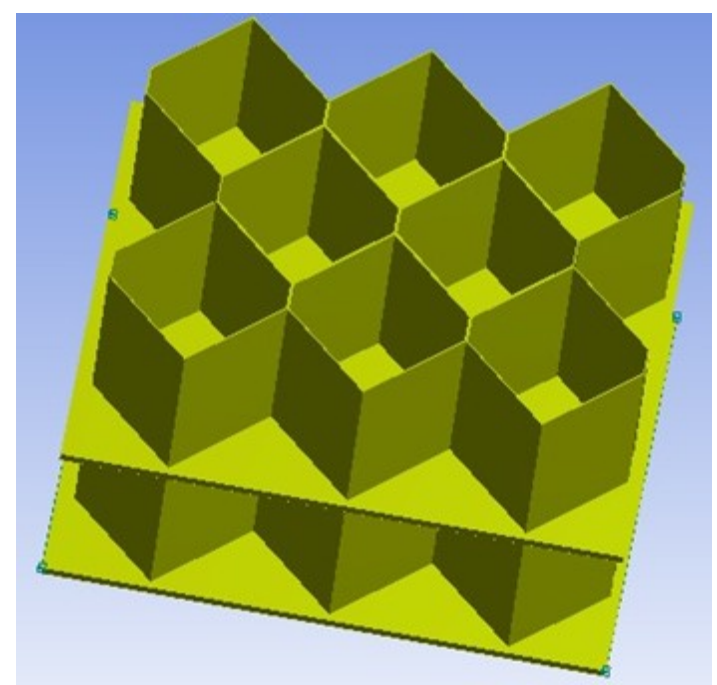

Fig.1 Model of double rhombic honeycomb core in ANSYS

\section{UNIT TYPE}

Although the model of honeycomb paperboard for layered structure, but based on its deformation is a complicated not easy convergence of the nonlinear process, so in this case, the Shell181 type element type. The Shell181 unit is in the form of four node plate type, each node has six degrees of freedom, the three is along the axis direction of translational degrees of freedom, the other three around the axis rotation degree of freedom. Honeycomb paperboard for viscoelastoplastic material, while the Shell181 unit form just also support such material. 


\section{MATERIAL PROPERTIES}

Two types of honeycomb paperboard used the same paper and core paper, but as a result of tissue paper and core paper fiber in different directions in the setting of material properties with two kinds of parameters, wherein the honeycomb paper core elastic modulus 0.36GPa, Poisson's ratio is 0.3 , tissue elastic modulus $0.76 \mathrm{GPa}$, Poisson ratio of 0.3 .

\section{GRID DIVISION}

The honeycomb grid has two kinds of methods: free mesh and the mapped mesh. Free mesh according to the unit of different types of automatic selection, free mesh model with fewer restrictions, but the method of dividing grid unit amount is larger, the grid is prone to distortion. Mapped mesh and free mesh compared by dividing the grid shape comparison rules, grid shape is not easy distortion, but the mapped mesh model more restrictions. Based on these two methods, their different characteristics, the model is used in the two meshing method combined with each other. Model grid partition after the graph is shown in figure 2 .

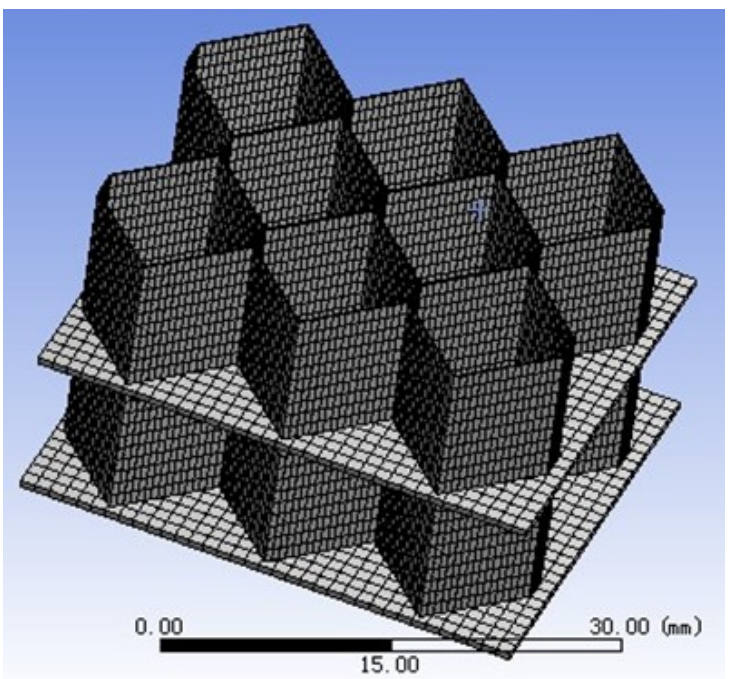

Fig.2 Double rhombic honeycomb paperboard model after meshing

\section{APPLIED LOAD}

The whole process of compressing the honeycomb cardboard is a complex nonlinear process. Nonlinear process includes three aspects: material nonlinearity and geometrical nonlinearity, state. Material nonlinearity is refers by the load displacement curves of honeycomb paperboard compression load, deformation and irreversible process. The geometric nonlinear refers to the honeycomb paperboard is compressed, geometry have a large deformation, deformation is not reversible. State nonlinear refers to when the honeycomb paperboard is compressed, and between the paper and paper core paper and paper due to the deformation of the material of some contact.
Research on the double rhombic honeycomb paperboard built model and double hexagonal honeycomb cardboard models are applied to different displacement load, the post-processing process from different displacement load compression diagram and stress distribution. This lists only after applying $15 \mathrm{~mm}$ displacement load $40 \mathrm{~mm}$ thick double rhombic honeycomb cardboard models of deformation and stress distribution, are shown in Figure 3 and figure 4.

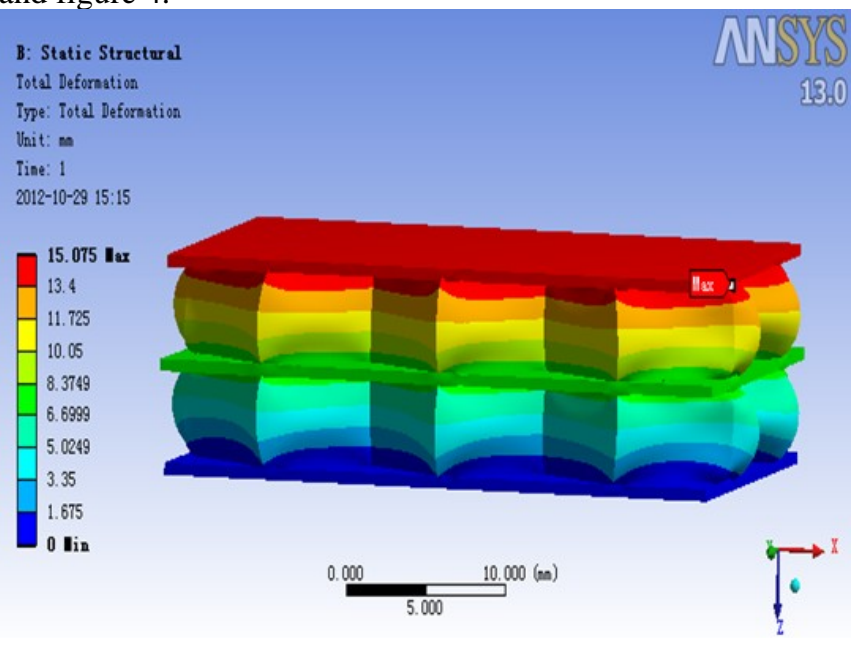

Fig.3 Deformation diagram of 15mm displacement

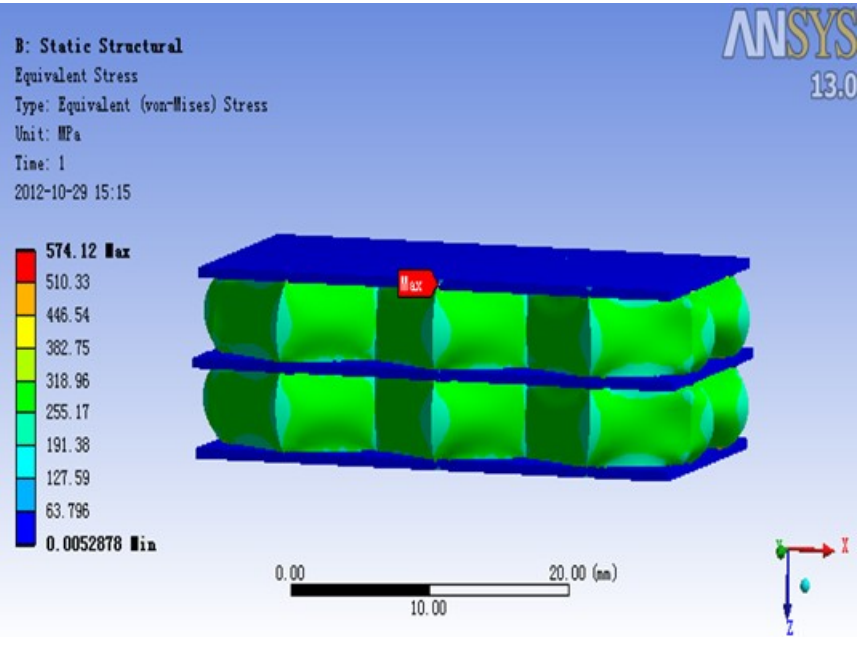

Fig.4 Displacement of $15 \mathrm{~mm}$ stress distribution diagram

\section{CONCLUSION}

Using the ANSYS Workbench software on different nest core, the same number and the same thickness of two kinds of honeycomb paperboard were simulated the stress analysis, in the process of simulation for the main model, material attribute selection, grid division and the applied load step, finally to obtain the different displacement under the load of honeycomb paperboard compression deformation, stress strain distribution map. 
The resulting in the same displacement load, double rhombic honeycomb cardboard the stress required to other types of honeycomb paperboard required stress to. The static compression test results. It shows that double rhombic honeycomb paperboard compression performance is better.

\section{REFERENC}

[1] Wenfeng Zhang, Anning Zhang .ANSYS8.0 based on the mechanical performance of honeycomb paperboard [J]. packaging engineering, 2006, 27 ( 2): 57-58

[2] Jianlin Peng, Zhihong Yin, Junjie Song. ANSYS based on static properties of honeycomb paperboard [J]. machine. 2007, 34 ( 10): 3133
[3] Houmin Li. The compression properties of honeycomb paperboard finite element analysis of [J]. packaging engineering, 2006, 24 ( 1): 13-15

[4] Wenfeng Zhang. mechanical performance of honeycomb paperboard study [D]. Anhui: Anhui University Of Science And Technology. 2006 2-3

[5] Yanfeng Guo, Jinghui Zhang, Wencai Xu. The honeycomb paperboard and the research on the cushion properties of [J]. packaging engineering. 2002.23 ( 5): 110-112

[6] Hailun Wang, Xing Zhang, etc. The core of honeycomb cardboard slotted flat compression strength effect of [J]. packaging Journal 2010.2 (4): 61-63 